\title{
Derivatives with respect to horizontal and vertical lifts of the Cheeger-Gromoll metric ${ }^{C G} g$ on Cotangent Bundle
}

\author{
HAŞIM ÇAYIR
}

\author{
Date of Receiving $\quad: \quad 27.12 .2016$ \\ Date of Revision : $\quad 22.02 .2017$ \\ Date of Acceptance : $\quad 23.02 .2017$
}

\begin{abstract}
In this paper, we define the Cheeger-Gromoll metric in the cotangent bundle $T^{*} M^{n}$, which is completely determined by its action on vector fields of type $X^{H}$ and $\omega^{V}$. Later, we obtain the covarient and Lie derivatives applied to the Cheeger-Gromoll metric with respect to the horizontal and vertical lifts of vector and kovector fields, respectively.
\end{abstract}

\section{Introduction}

Cheeger-Gromoll metric was defined by Cheeger and Gromoll in [3] and the explicit formula for this metric was given by Musso and Tricerri in [15]. The Levi-Civita connection of ${ }^{C G} g$ and its Riemannian curvature tensor are calculated by Sekizawa in [20] and corrected by Gudmundsson and Kappos in [11]. In [19] Salimov and Kazimova studied geodesics of the Cheeger-Gromoll metric on the tangent bundle. The similar metric in theoritical physics has been obtained by Tamm (Nobel Laureate in Physics for the year 1958, see [21]). The geometry of the tangent bundle equipped with Cheeger-Gromoll metric is well known and intensively studied (see for example $[9,10,11,14,18,19])$. In [1] Ağca and Salimov investigate curvature properties and geodesics on the cotangent bundle with respect to the Cheeger-Gromoll metric.

The tangent bundles of differentiable manifolds are very important in many areas of mathematics and physics. Cotangent bundle is dual of the tangent bundle. Because of this duality, some of the geometric results are similar to each other. The most significant difference between them is construction of lifts (see [22] for more details). In this paper, we define the Cheeger-Gromoll metric in the cotangent bundle $T^{*} M^{n}$, which is completely determined by its action on vector fields of type $X^{H}$ and $\omega^{V}$. Later, we obtain the covarient and Lie derivatives applied to the Cheeger-Gromoll metric with respect to the horizontal and vertical lifts of vector and kovector fields, respectively.

2010 Mathematics Subject Classification. 15A72, 47B47, 53A45, 53C15.

Key words and phrases. Covarient Derivative, Lie Derivative, Cheeger-Gromoll metric, Horizontal Lift, Vertical Lift.

Communicated by: A.A. Salimov 